\title{
¿Cuál es el rumbo futuro de la Unión Europea? El debate en torno a los diferentes caminos posibles
}

María Victoria Álvarez*

La actual Unión Europea (UE), lejos de haber completado una "unión", está lidiando con varias crisis al mismo tiempo, en ámbitos como la economía, las finanzas, el empleo, las migraciones y la seguridad, y hasta enfrentando fuertes cuestionamientos a su legitimidad política.

Aunque parezca increíble, la hazaña de haber conseguido que franceses y alemanes cooperaran conjuntamente en la segunda posguerra y erigieran un proyecto integracionista en común, se ha visto amenazada en los últimos años por problemas que hacen temblar al bloque hasta sus cimientos.

Pero además, en los últimos meses, los indicios de que las crisis empezaban a ser superadas se ven desafiados por nuevos elementos disruptivos. La decisión del Reino Unido de abandonar la UE, las iniciativas independentistas en algunos Estados miembros y el accionar del (nuevo) gobierno de Estados Unidos hacia los socios europeos se han convertido en las más recientes vicisitudes.

En este marco, en marzo de 2017 la Comisión Europea publicó el Libro Blanco sobre el futuro de Europa: Reflexiones y escenarios para la Europa de los Veintisiete en 2025 mientras se preparaba para celebrar el $60^{\circ}$ aniversario de la firma de los Tratados de Roma ${ }^{1}$. El documento destacaba, en un tono ligeramente triunfalista, el logro de siete décadas de paz y una UE ampliada de 500 millones de ciudadanos que viven en una de las economías más prósperas del mundo, pero advertía que, al mismo tiempo, la UE debía mirar hacia el futuro y perfilar la visión de su porvenir con veintisiete Estados miembros en lugar de veintiocho. Así, el documento describe los principales desafíos y oportunidades que encara Europa en el presente y futuro próximos, presentando cinco escenarios sobre cómo podría evolucionar el bloque de aquí a 2025 dependiendo del camino que decida tomar.

Entre los factores que se presentan como desafíos para el futuro de la UE el Libro Blanco implicaba a la debilitada posición de Europa en el mundo en cuanto a población y poder económico en términos relativos, un contexto mundial cada vez más tenso, una sociedad europea que todavía no termina de recuperarse de la crisis económica y financiera, el incremento de las amenazas y la inquietud por la seguridad y las fronteras, así como la creciente desafección por la política y las instituciones por parte de los ciudadanos.

En base al Libro Blanco, este breve artículo de opinión analiza los cinco escenarios propuestos, sus méritos y desventajas y sus implicancias para el rumbo futuro del proceso de integración europea, rumbo que se percibe incierto en la actualidad, tal vez como nunca antes en la historia del bloque. Atendiendo a que, en su presentación del Libro Blanco en el Parlamento

\footnotetext{
* Doctora en Relaciones Internacionales y docente, Facultad de Ciencia Política y Relaciones Internacionales, Universidad Nacional de Rosario (Argentina). Correo electrónico: maria.alvarez@fcpolit.unr.edu.ar

${ }^{1}$ COMISIÓN EUROPEA, Libro Blanco sobre el Futuro de Europa. Reflexiones y escenarios para la Europa delos Veintisiete en 2025. Disponible en https://ec.europa.eu/commission/sites/betapolitical/files/libro_blanco_sobre el futuro_de europa es.pdf. Consulta: 25 de noviembre de 2017
} 
Europeo, el Presidente de la Comisión Europea, Jean-Claude Juncker, aseguró que era necesario abrir un debate "difícil" y "real" sobre la UE a veintisiete ${ }^{2}$, el trabajo incluye una breve reflexión crítica en su apartado final, para intentar responder si (y hasta dónde) el documento impulsa el debate difícil y real que marcaba Juncker.

\section{Escenario 1: Seguir igual}

Históricamente, la integración europea se desarrolló en base a la idea de avanzar en aquellas cuestiones de menor visibilidad y menos polémicas dando "pequeños pasos". La integración gradual y acumulativa de sectores concretos de la actividad económica se tradujo en el establecimiento, primero, de un mercado común del carbón y del acero, para avanzar, posteriormente, con la Comunidad Económica Europea y el Euratom. Una vez conseguida la meta de la unión aduanera y de políticas comunes para los sectores involucrados, los países europeos lograron alcanzar el mercado común para las mercancías, servicios y capitales, incluida la libre circulación de personas.

Al constituir a la UE, el Tratado de Maastricht de 1992 suponía una nueva etapa en el proceso de creación de una "unión cada vez más estrecha entre los pueblos de Europa", manifestada en sus proyectos de Unión Económica y Monetaria (UEM), introducción de una Política Exterior y de Seguridad Común (PESC) y gestación de una ciudadanía europea. Ésta ha sido la última maniobra concreta en pos de alcanzar una unión política como meta final de la integración europea, pero el método de los "pequeños pasos" parece agotarse.

El primer escenario, entonces, es el de seguir adelante o continuar igual. Los 'insiders' en Bruselas se refieren a él como el "modelo de Bratislava", en referencia a la Declaración de Bratislava, de septiembre de 2016, en la que fue la primera reunión de todos los jefes de Estado y de Gobierno de la UE sin el Reino Unido ${ }^{3}$. Allí los Veintisiete aceptaban que la UE no es perfecta, pero aseguraban que es el mejor instrumento a disposición para afrontar los desafíos que sufre. Subyace entonces la idea de seguir básicamente como hasta ahora: el mismo modelo de integración imperfecto tal cual lo conocemos hoy en día, con sus ventajas y desventajas.

Es el escenario más realista, sin más integración ni menos, algo que supone seguir "dando resultados concretos". Sin Reino Unido, en una UE de veintisiete Estados miembros, con este escenario se preserva la unidad del bloque, aunque "ésta puede verse cuestionada en caso de conflictos importantes", advierte el documento.

Es evidente que la UE puede seguir como hasta ahora, sin grandes cambios, pero esa opción conlleva el riesgo de que en poco tiempo estemos planteando futuros escenarios para un proceso de integración nuevamente en crisis. Seguir igual equivale a continuar con la dinámica de "politics as usual", donde el proceso decisorio permanece siendo difícil de entender, y la capacidad de obtener resultados no siempre se corresponde con las expectativas de los europeos: una lógica que difícilmente logre cerrar la brecha existente entre las instituciones europeas y los ciudadanos, y que se ha acrecentado en los últimos años.

\section{Escenario 2: Solo el mercado único}

Las dificultades para avanzar hacia un sistema bancario unificado o una política europea común de asilo constituyen claras manifestaciones actuales de que la integración posee

\footnotetext{
2 Juncker pone sobre la mesa reducir la UE a un simple mercado único, La Vanguardia, 1 de marzo de 2017. Disponible en http://www.lavanguardia.com/internacional/20170301/42430755023/junckerdeshacer-integracion-europea.html Consulta: 11 de diciembre de 2017

${ }^{3}$ CONSEJO EUROPEO, Declaración y hoja de ruta de Bratislava, 16 de septiembre de 2016. Disponible enhttp://www.consilium.europa.eu/es/press/press-releases/2016/09/16/bratislava-declaration-androadmap/. Consulta: 30 de noviembre de 2017
} 
inconvenientes a la hora de prosperar más allá de ciertos límites, y que los Estados son reticentes frente a la puesta en común bajo la égida de la UE de las pocas áreas de política que aún subsisten en la esfera nacional.

La UEM, hoy en funcionamiento en diecinueve de los (aún) veintiocho Estados miembros, se encuentra en graves problemas, y si bien la situación no es tan seria como hace unos años, hay Estados miembros que siguen acusando serios inconvenientes en términos económicos y sociales. Incluso algunos especulan que en Europa, más allá de la fase de mercado común, la integración tiene importantes dificultades para subsistir.

Entonces, el segundo escenario vaticina que la UE de los Veintisiete se centrará gradualmente en el mercado único, al no alcanzar acuerdos los Estados miembros en un número creciente de ámbitos. De hecho, recoge la posibilidad de que los países terminen limitando la UE a un mercado único circunscripto a la libre circulación de bienes y capitales (pero sin garantizar plenamente la libre circulación de personas y servicios).

El Libro Blanco, de esta manera, predice como segunda posibilidad para el futuro de la UE algo que hasta ahora era un tema completamente tabú para Bruselas: olvidarse de la integración progresiva y dar marcha atrás en parte de los avances ya hechos en las últimas décadas.

Esta opción supone centrarse en el ámbito económico y renunciar a una política común en refugiados, inmigración o asuntos sociales. Implica una lenta "deconstrucción" del proyecto actual para centrarse en lo que fue la Comunidad Económica Europea.

Es la alternativa de "deshacer Europa" y aunque principalmente para la Comisión esta opción es aborrecible, "debe evaluarse" . Ahora mismo tampoco es la posición preferida por los Estados miembros. Pero podría llegar a ser una opción para salvar a la integración si las diferencias entre los Estados miembros y entre éstos y las instituciones europeas se incrementan, si no se logran consensos, o hay resultados electorales negativos para la UE actual.

En línea con este escenario, son diversos los analistas que creen que la integración llegó a un punto no sólo donde no puede seguir avanzando sino que sería preferible que retroceda. El sociólogo Wolfgang Streeck, por ejemplo, sostiene que el objetivo a perseguir no es la unión federal (inviable y, en principio, indeseable para el autor), sino más bien el restablecimiento de las soberanías nacionales. En términos económicos, esto significa desmontar la UEM, regresar a tipos de cambio flexibles y así, a la posibilidad de utilizar la devaluación de la moneda como un instrumento fundamental para abordar los desequilibrios macroeconómicos (Pistone, 2013: 130).

Ya en los años setenta, el autor neofuncionalista Philippe Schmitter advertía que la integración no siempre (ni necesariamente) mantiene como trayectoria una progresión lineal y automática. Schmitter admitía que los actores involucrados en la integración podían no sólo avanzar sino también elegir entre opciones alternativas, como el spillback, que implicaba un retroceso en el nivel y el alcance de la autoridad supranacional (Schmitter, 2004).

En este caso, los Estados miembros optan por renunciar al objetivo original de la integración, escogiendo estrategias "desintegrativas". Como resultado, la toma de decisiones "puede ser más fácil de entender, pero la capacidad de actuar de manera colectiva será limitada", advierte el Libro Blanco, que añade que esto puede "aumentar la brecha entre las expectativas (ciudadanas) y los resultados a todos los niveles". Estas estrategias son, por supuesto, costosas sobre todo luego de siete décadas de proceso, e implican el retorno a vinculaciones de tipo bilateral para una gran variedad de ámbitos de política.

\footnotetext{
${ }^{4}$ Moscovici: la opción de 'deshacer' Europa está sobre la mesa y debe evaluarse, EFE, 1 de marzo de 2017. Disponible enhttps://www.efe.com/efe/espana/mundo/moscovici-la-opcion-de-deshacer-europaesta-sobre-mesa-y-debe-evaluarse/10001-3194276Consulta: 1 de diciembre de 2017
} 


\section{Escenario 3: Los que desean hacer más, hacen más}

La UE de los Veintisiete sigue funcionando como en la actualidad, pero permite a los Estados miembros que lo deseen una mayor colaboración en ámbitos específicos, como los asuntos sociales, la defensa, o la seguridad interior, a través de la cooperación reforzada, un mecanismo que existe en la legislación europea y que nació como consecuencia de la diversidad de sensibilidades, objetivos y prioridades dentro de la UE.

La cooperación reforzada constituye una opción "a la carta" para avanzar, a diferencia de lo que hoy ya existe y que son las cláusulas de opt-out, que permiten a los Estados miembros quedar al margen de algunas políticas concretas ${ }^{5}$. Los Estados miembros que desean integrarse más para avanzar (y no para eximirse con cláusulas derogatorias), entonces, pueden hacerlo.

El escenario de las "dos velocidades" o de las "múltiples velocidades" no es precisamente nuevo, como hemos mencionado. Los Tratados lo permiten. La cooperación reforzada fue regulada por primera vez por el Tratado de Ámsterdam (1997) y simplificada por el Tratado de Niza (2001) ${ }^{6}$. Además, es políticamente viable, como lo demuestran iniciativas anteriores tales como el Espacio de Libertad, Seguridad y Justicia, la conformación de la zona euro, y diferentes acciones en el ámbito de la Política Exterior y de Seguridad Común.

Las "múltiples velocidades" suponen que no hay un avance en la integración a Veintisiete, sino en diferentes bloques. Es decir, puede haber coaliciones y grupos de países que se ponen de acuerdo para profundizar en un ámbito de política, pero lo decidido no es obligatorio para los demás. De esta manera, en temas muy concretos, si se constata que no hay unidad absoluta, algunos seguirían adelante y otros no. Una fórmula que puede colmar las aspiraciones de quienes tienen cosmovisiones completamente diferentes y hasta enfrentadas dentro de la familia europea.

Esto es lo que ha ocurrido con veintitrés ${ }^{7}$ de los veintiocho países del bloque (a excepción de Malta, Dinamarca, Irlanda, Portugal y Reino Unido), quienes, el 11 de diciembre de 2017, dieron el visto bueno a la creación de una Cooperación Permanente Estructurada en Defensa (PESCO, en sus siglas en inglés), que permitiría, a partir de algunos proyectos iniciales, ir poniendo los pilares de una futura defensa común del bloque en paralelo a la pertenencia a la Organización del Tratado del Atlántico Norte (OTAN) de la mayoría de los países de la UE.

Según el Presidente de la Comisión, Jean-Claude Juncker, el modelo de "los que desean más, hacen más" es probablemente el escenario más realista de todos. Inclusive, sugirió a partir de este modelo la posibilidad de una especie de "re-concepción" de la UE que implicaría la existencia de un grupo pequeño de miembros de la UE y diferentes niveles de pertenencia para otros países que no están preparados para (o no desean) ser miembros plenos.

En particular, Juncker propuso un "diseño estructurado, abierto a todos" en un formato que comprendería a algunos miembros centrales rodeados por una "órbita" externa de países menos involucrados. Esta estructura más laxa podría incluir a Reino Unido una vez que salga del bloque, por ejemplo, o incluso a Turquía, que ha estado persiguiendo la adhesión a la UE por años ${ }^{8}$.

\footnotetext{
${ }^{5}$ Los británicos, por ejemplo, no forman parte del Acuerdo Schengen, ni del Espacio de Libertad, Seguridad y Justicia, ni tampoco aplican la Carta de los Derechos Fundamentales de la UE, en lo que constituía su tradicional práctica de buscar "salidas parciales" ("partial exits") dentro de la UE.

${ }^{6}$ Actualmente, las "Cooperaciones reforzadas" se encuentran normadas en el Tratado de la Unión Europea (TUE), en su Título IV (artículo 20), y el Tratado de Funcionamiento de la Unión Europea (TFUE), en su Título III (artículos 326 a 334).

${ }^{7}$ Diecinueve de los cuales forman parte de la OTAN.

${ }^{8}$ Juncker envisions EU overhaul, roles for post-Brexit UK and Turkey, Deutsche Welle, 24 de febrero de 2017. Disponible en http://www.dw.com/en/juncker-envisions-eu-overhaul-roles-for-post-brexit-uk-andturkey/a-37697208. Consulta: 5 de diciembre de 2017
} 
No debemos confundir, sin embargo, las declaraciones del Presidente de la Comisión con la cooperación reforzada tal como se concibe en los Tratados: este mecanismo está pensado para fortalecer la cooperación entre Estados miembros, mientras que la versión de Juncker viene a proveer una posible solución para la vinculación entre Estados miembros y terceros países (ya sea Estados que no forman parte de la UE como Turquía o Estados que abandonarán la UE, como Reino Unido).

\section{Escenario 4: Hacer menos pero de forma más eficiente}

La UE de los Veintisiete centra su atención en aumentar y acelerar los logros en los ámbitos de actuación prioritarios que ha elegido, mientras que interviene menos en los que se percibe que no aporta valor añadido.

El cuarto escenario que propone el Libro Blanco aparenta ser sencillo, pero en realidad es el más complicado de todos: que los Estados miembros centren su atención y sus recursos en un número limitado de ámbitos de política. Los Estados miembros deberían consensuar en qué áreas hay voluntad para avanzar y sólo en ellas profundizar la integración al máximo. Esto implicaría ceder atribuciones a las instituciones europeas en aquellas áreas consensuadas, y "renacionalizar" las competencias en todas las demás.

Si lo pensamos en términos del binomio "integración-desintegración”, este escenario provocaría una situación mixta o intermedia. Habría desintegración en algunas áreas - en el sentido de que las competencias volverían a ser nacionales - e integración a nivel supranacional en otras.

La viabilidad de la "renacionalización" de políticas comunitarias, es decir, la reducción de competencias atribuidas a la UE, al momento de decidir la revisión de los $\operatorname{tratados}^{9}$ fue introducida por la última reforma de los tratados constitutivos, el Tratado de Lisboa. Este tratado fue el que abrió otra puerta a la reversibilidad del proceso integrativo, introduciendo la posibilidad de la retirada de un Estado ${ }^{10}$.

La desintegración, entonces, incluye la probabilidad de que el proceso retroceda en términos de políticas o áreas integradas o del nivel de autoridad de las instituciones centrales (el spill-back antes mencionado), como podría ocurrir en caso de que se fragmente la UEM, y que algún Estado de la zona euro regrese a una política monetaria autónoma y una moneda nacional. De seguir por esa línea, nos acercaríamos al modelo número dos.

Pero, en este escenario, como contrapartida de la retirada de la UE de ciertos ámbitos de política, los Estados miembros se comprometen a acelerar y profundizar la integración en otros. Por un lado sería un avance histórico, que llevaría al nivel europeo lo que todavía está fragmentado ahora. Por el otro, un retroceso: la confirmación del hecho de que los países miembros no están interesados ni tienen la voluntad para profundizar la integración hasta el final.

Especialmente este escenario se presenta como riesgoso políticamente, ya que dar marcha atrás en aquellos ámbitos "donde se percibe que la UE tiene un limitado valor añadido o incapaz de cumplir sus promesas", constituye una opción que plantea dudas sobre el tipo de áreas que quedarían fuera o sobre cómo sería el proceso de toma de decisiones para poder llevar adelante esa decisión ${ }^{11}$.

\footnotetext{
${ }^{9}$ Art. 48.2 del Tratado de la Unión Europea, TUE

${ }_{11}^{10}$ Art. 50 del Tratado de la Unión Europea, TUE

${ }^{11}$ Algunas áreas, según el Libro Blanco, podrían ser la innovación, el comercio, la seguridad, la migración, la gestión de las fronteras y la defensa, I+D o la descarbonización y la digitalización a escala de la UE.
} 


\section{Escenario 5: Hacer mucho más conjuntamente}

Los Estados miembros deciden compartir más competencias, recursos y toma de decisiones en todos los ámbitos. Es decir, la cooperación entre los Estados miembros va más lejos que nunca en todas las áreas de política y del mismo modo, la zona del euro se consolida. Las decisiones se adoptan con mayor rapidez a nivel europeo y se aplican con celeridad a nivel nacional.

El último escenario del Libro Blanco es el extremo opuesto al escenario número dos, de centrarse sólo en el mercado único. La quinta opción es, justamente, el "sueño federal" de los padres fundadores, de Monnet, Schuman y Adenauer.

Los Tratados de Roma significaron el triunfo de la dinámica neofuncionalista de spillover (desbordamiento), concepto que aborda la expansión horizontal (intersectorial) del proceso de integración. Se trataba, en definitiva, de iniciar un proceso en el que la paulatina integración económica fuera allanando el camino hacia el objetivo final de lograr la unión política, meta que se fijaba a largo plazo.

Este modelo supone reducir al mínimo las competencias soberanas de los Estados miembros y ceder al máximo a la UE, es decir, buscar una respuesta europea a todo, sin recelos nacionales ni luchas por porciones de soberanía. Sin embargo, esta opción es prácticamente impensable. Según algunos, Europa está más cerca de quedarse únicamente como mercado único, es decir, el escenario número dos, que acabar siendo los Estados Unidos de Europa.

Este escenario es aquél en el cual se avanza en áreas de política hasta donde nunca se había llegado antes. Lo que debería ocurrir es que en cada ámbito, ya sea comunitario o no, se diera un progreso hacia la profundización de la integración. La reciente firma de un acuerdo sobre el PESCO para aunar esfuerzos y colaborar estrechamente en temas de seguridad y defensa, como hemos mencionado anteriormente, podría servir como ejemplo de un paso en esta dirección.

Si bien, como indicábamos anteriormente, esta iniciativa constituye una muestra de la Europa de las "dos velocidades", ya que cinco países decidieron quedar fuera, los firmantes esperan que el resto, bien por rendirse a la evidencia, bien por miedo a quedarse al margen, acaben sumándose en el futuro.

Si ello se cumple, es decir, si la UE logra sacar adelante éste y otros temas a veintiocho (o a veintisiete dentro de unos pocos años), y consigue que no haya países empeñados en bloquear dichos avances, nos vamos acercando al modelo quinto del Libro Blanco. Un escenario que sustrae a Europa de la inacción, conducidos por la impronta integracionista de Alemania y Francia.

\section{Algunas consideraciones finales}

La política fiscal, la austeridad, la crisis de refugiados, la gestión de las fronteras, el Brexit, o los gobiernos euroescépticos en Polonia o Hungría han dividido a la UE en los últimos años. Los Veintisiete necesitan algo para aferrarse para salir unidos y reforzados una vez que Reino Unido efectivice su salida.

El objetivo del Libro Blanco sobre el futuro de Europa presentado por la Comisión Europea en marzo de 2017 era exhibir una serie de escenarios sobre cómo podría evolucionar Europa desde ahora hasta 2025. Buscaba abrir un debate que debía ayudar a centrar la reflexión y encontrar nuevas respuestas a la vieja pregunta sobre qué tipo de futuro quieren los europeos para ellos, para sus hijos y para la Unión.

Luego de haber realizado un sucinto análisis de los cinco escenarios, sus ventajas y desventajas y sus implicancias para el porvenir de la UE, estamos en condiciones de realizar algunas consideraciones finales. En primer lugar, el Libro Blanco constituye un interesante 
(aunque tímido) intento por ordenar el debate y la reflexión en torno a las posibles rutas que puede tomar la integración, pero no trasciende el análisis (y el lenguaje) tecnocrático, opaco y economicista propio de los documentos de la Comisión Europea.

En segundo lugar, el documento da cuenta de que la UE está en serios problemas y de que no hay señales de que los retos de Europa vayan a disminuir. Sin embargo, más allá de este diagnóstico, el documento se queda a mitad de camino. No aborda con total franqueza problemas dolorosos para los ciudadanos europeos como el desempleo, el desempleo juvenil, la desigualdad, la precariedad laboral y social o la situación humanitaria de los refugiados. Tampoco intenta esbozar soluciones para problemas graves para la UE como el desapego de los ciudadanos, el auge de fuerzas populistas y euroescépticas, especialmente de extrema derecha, o la ausencia de una identidad europea. Es posible que estas cuestiones estén todas estrechamente relacionadas y se extraña una determinación más categórica de la UE para abordarlas.

En tercer lugar, algunos de los escenarios, especialmente el segundo y el cuarto, reconocen sin tapujos la eventualidad de que la integración inicie algún tipo de desintegración de mayor o menor intensidad. El Libro Blanco cristaliza, entonces, la necesidad de plantearse seriamente la posibilidad de desintegración, y ello, creemos, es un signo de que las instituciones europeas poseen alguna capacidad de autocrítica y reflexión genuina.

Ha sido usual en los últimos años el llamado al debate y a la reflexión, especialmente cuando la complejidad de las circunstancias conduce a los líderes europeos a la perplejidad. Ya en 2001 los Estados miembros se planteaban múltiples interrogantes respecto al futuro de Europa, a través de la Declaración de Laeken ${ }^{12}$. En ese momento, los gobiernos estaban preocupados por la inminente ampliación hacia Europa central y oriental. Entre los desafíos se incluía aproximar las instituciones europeas a los ciudadanos, que no siempre percibían la relación entre los objetivos y la actuación cotidiana de la Unión. Posteriormente, en 2005, luego de los referendos negativos en Francia y Países Bajos sobre la Constitución Europea, los Estados miembros decidieron realizar una pausa para la reflexión ${ }^{13}$. Según el Consejo Europeo, los referendos fallidos ponían de manifiesto preocupaciones e inquietudes de los ciudadanos que había que tener en cuenta. Por ello, la pausa para la reflexión debía dedicarse a fomentar el debate con los ciudadanos, y tomar nota de qué Europa quería.

Como en 2001 y 2005, lo más probable que ocurra es que habrá pausa en el proceso político, pero reflexión, poca. Si la historia se repite, como suele ocurrir, el debate y la reflexión a la que convoca el Libro Blanco de la Comisión quedarán, como en ocasiones anteriores, en meras intenciones políticas. Luego los hechos demuestran que los gobiernos y las instituciones europeas caen en una apatía generalizada para desarrollar ese diálogo con los ciudadanos.

En definitiva, la publicación de documentos como éste intenta mostrar una acción de impulso y de iniciativa por parte de las instituciones europeas. Sin embargo, más allá de un diagnóstico bastante acertado y la introducción de la desintegración como una opción razonable para la futura trayectoria del proceso, el Libro Blanco vuelve a caer en el error de no profundizar en cuestiones como la igualdad, la justicia, la solidaridad y el bienestar, valores caros a los ciudadanos, e insiste en opciones economicistas simples y llanas que impiden reformas reales y poderosas para que los europeos vuelvan a confiar en la UE y a creer en ella como la llave para su prosperidad, en el futuro, pero también en el presente.

\footnotetext{
12 CONSEJO EUROPEO, Conclusiones de la Presidencia -Anexo I Declaración de Laeken sobre el futuro de la Unión Europea. Laeken, 14 y 15 de diciembre de 2001. Disponible en http://europa.eu/rapid/press-release_PRES-01-902_es.pdf. Consulta: 11 de diciembre de 2017

${ }^{13}$ CONSEJO EUROPEO, Declaración de los Jefes de Estado y de Gobierno de los Estados miembros de la Unión Europea sobre la ratificación del Tratado por el que se establece una Constitución para Europa, Bruselas, 16 y 17 de junio de 2005. Disponible en http://europa.eu/rapid/press-release_DOC-05-3 es.pdf Consulta: 18 de diciembre de 2017
} 
Cuadernos de Política Exterior Argentina (Nueva Época), 126, julio-diciembre 2017, pp. 87-94 ISSN 0326-7806 (edición impresa) - ISSN 1852-7213 (edición en línea)

\section{Bibliografía}

PISTONE, S. (2013). The Debate in Germany on Democracy and European Unification: a Comparison of the Positions of Habermas and Streeck, The Federalist, LV, pp. 126-135

SCHMITTER, P. C. (2004). Neo-neofunctionalism, en WIENER, A.; DIEZ, T., European integration theory, Oxford University Press, Oxford 